\title{
Alternative Primary Structures in the Transmembrane Domain of the Chicken Erythroid Anion Transporter
}

\author{
JOHN V. COX AND ELIAS LAZARIDES* \\ Division of Biology, California Institute of Technology, Pasadena, California 91125
}

Received 13 October 1987/Accepted 1 December 1987

\begin{abstract}
Isolation and characterization of the chicken erythroid anion transporter (band 3) cDNA clone, pCHB3-1, revealed that the chicken erythroid band 3 polypeptide is 844 amino acids in length with a predicted mass of 109,000 daltons. This polypeptide is composed of a hydrophilic N-terminal cytoplasmic domain and a hydrophobic C-terminal transmembrane domain. The $\sim 90 \mathrm{~N}$-terminal amino acids of the human and murine erythroid band 3 polypeptides are absent in the predicted sequence of the chicken erythroid band 3 polypeptide. The absence of this very acidic $\mathrm{N}$-terminal region is consistent with the lack of binding of glyceraldehyde-3-phosphate dehydrogenase to chicken erythroid band 3, as well as the relatively basic isoelectric point observed for this molecule. The remainder of the cytoplasmic domain shows little similarity to the cytoplasmic domain of the murine and human erythroid band 3, with the exception of the putative ankyrin-binding site, which is highly conserved. In contrast, the transmembrane domain of the chicken band 3 polypeptide is very similar to that of the murine erythroid and human nonerythroid band 3 polypeptides. The transmembrane domain contains 10 hydrophobic regions that could potentially traverse the membrane 12 to 14 times. In addition, a variant of chicken erythroid band 3, pCHB3-2, was cloned in which one of the hydrophobic regions of pCHB3-1 is lacking. The transcript complementary to pCHB3-2 accumulated in chicken erythroid cells in a similar manner as the transcript complementary to $\mathrm{pCHB3-1}$ during embryonic development. This is the first example of a transporter protein or ion channel with alternative primary structures in its membrane-spanning segments.
\end{abstract}

The anion transporter, also known as band 3 , is the predominant integral membrane protein of mammalian (44) and avian $(12,22)$ erythroid cells. Biochemical and physiological studies have indicated that the band 3 polypeptide possesses multiple functions that are segregated into two distinct domains. The $\mathrm{C}$-terminal membrane-spanning region of band 3 mediates anion transport, primarily the one-forone exchange of chloride for bicarbonate (28). This rapid, electroneutral exchange process increases the $\mathrm{CO}_{2}$-carrying capacity of the blood as well as stabilizes the extracellular $\mathrm{pH}$ (14). The $\mathrm{N}$-terminal cytoplasmic region of band 3 provides the membrane attachment site for the erythrocyte membrane cytoskeleton through its interaction with ankyrin $(3,21)$, protein $4.1(40)$, and protein $4.2(11)$. The extreme $N$ terminus of the cytoplasmic domain also possesses highaffinity binding sites for hemoglobin (10) and the glycolytic enzymes aldolase (38) and glyceraldehyde-3-phosphate dehydrogenase $(47,52)$, although the latter is absent in both the murine (30) and chicken (22) band 3 polypeptide. In addition to the functionally analogous erythroid band 3 polypeptides that have been described in many species, immunological studies have demonstrated band 3-like polypeptides in a variety of nonerythroid tissues $(12,16,27)$.

The recent isolation and characterization of cDNA clones for the murine erythroid (30) and human nonerythroid (15) band 3 polypeptides have revealed several features of band 3 that are conserved. The predicted sequences of the transmembrane domains of the murine erythroid and human nonerythroid band 3 polypeptides are very similar to each other and to the transmembrane regions of the human erythroid band 3 polypeptide that have been sequenced $(8$, 35). The hydropathy profile of these polypeptides reveals 10 very hydrophobic regions that have been proposed to span

\footnotetext{
* Corresponding author.
}

the membrane as many as 12 times (30). Characterization of the murine band 3 gene (29) has indicated that each of these hydrophobic membrane-spanning segments roughly corresponds to an exon of the band 3 gene. The predicted sequence of the cytoplasmic domain of the murine erythroid band 3 polypeptide is also very similar to those regions of the cytoplasmic domain of the human erythroid band 3 polypeptide that have been sequenced (26), although the sequence has diverged at the extreme $\mathrm{N}$ terminus where the binding sites for the glycolytic enzymes are located in the human molecule $(38,47)$. Both the murine and human erythroid band 3 polypeptides have relatively little similarity to the predicted sequence of the cytoplasmic domain of the human nonerythroid band 3 polypeptide. However, the region of the cytoplasmic domain where the ankyrin-binding site has tentatively been placed (34) exhibits considerable homology among all the band 3 polypeptides, suggesting that this high-affinity binding site is conserved in erythroid and nonerythroid cell types.

We describe here the isolation and characterization of a cDNA clone, pCHB3-1, for chicken erythroid band 3. These studies revealed that the transmembrane domain of the chicken erythroid band 3 polypeptide is greater than $70 \%$ identical to the membrane-spanning region of the band 3 polypeptides from other species. The hydropathy profile suggests that chicken erythroid band 3 traverses the membrane 12 to 14 times, in agreement with previous results $(15$, 30). Furthermore, many of the amino acids implicated in anion transport through drug binding studies are conserved in the chicken erythroid band 3 polypeptide. Sequence analysis in conjunction with in vitro transcription and in vitro translation studies indicates that the $\sim 90 \mathrm{~N}$-terminal amino acids of human (26) and murine (30) erythroid band 3 are absent in chicken erythroid band 3 . The lack of this cytoplasmic region is consistent with the absence of binding 
of chicken erythroid band 3 to glyceraldehyde-3-phosphate dehydrogenase (22). The remainder of the cytoplasmic domain is only $\sim 40 \%$ similar to the cytoplasmic domain of murine erythroid band 3 , although the region where the ankyrin-binding site has tentatively been placed is highly similar, suggesting that this site in the band 3 polypeptide is conserved in all species. Finally, an additional band 3 cDNA, pCHB3-2, was isolated that encodes a variant chicken erythroid band 3 polypeptide lacking one of the putative transmembrane-spanning segments found in pCHB3-1. S1 nuclease analysis indicated that the RNA complementary to pCHB3-2 accumulates in erythroid cells during development in a manner similar to that previously reported for the RNA complementary to pCHB3-1 (13). Interestingly, the boundaries of the region that is absent in the RNA complementary to pCHB3-2 almost exactly correspond to two of the intron-exon splice junctions of the murine band 3 gene (29). This suggests that the equivalent of murine exon 15 (29) is removed from this transcript by alternative splicing. This represents the first report of a transporter protein or ion channel with an alternative primary structure in its membrane-spanning segments.

\section{MATERIALS AND METHODS}

Isolation and characterization of band 3cDNA clones. A $\lambda$ gt11 cDNA library constructed with poly(A) ${ }^{+}$RNA from erythroid cells isolated from 15-day-old chicken embryos (37) was screened with the nick-translated (42) insert of the previously described band 3 cDNA clone, p3(9-1) (12). Filters were prepared and hybridized as described previously (4), and cDNA inserts from positive clones were isolated and subcloned into the pGEM-3 vector (Promega Biotec, Madison, Wis.). The restriction endonuclease sites within the cDNA inserts were determined by single and double digests with various restriction endonucleases, and the digestion products were resolved on agarose gels.

DNA sequencing. The cDNA inserts from band 3-positive clones were also subcloned into $\mathrm{M} 13 \mathrm{mp} 19$, and both strands were sequenced by the dideoxy chain termination method (43). Oligonucleotide primers for sequencing reactions and primer extension analysis were synthesized on an automated DNA synthesizer at the California Institute of Technology microchemical facility.

Primer extension analysis. An oligonucleotide primer corresponding to nucleotides -690 to -671 of pCHB3-1 (Fig. 1) was end labeled with $\mathrm{T} 4$ polynucleotide kinase in the presence of $\left[\gamma^{-32} \mathrm{P}\right]$ ATP. A 10 -pmol sample of this ${ }^{32} \mathrm{P}$-labeled primer was hybridized to $5 \mu \mathrm{g}$ of total RNA from erythroid cells isolated from 5-day-old chicken embryos. The primer was extended with reverse transcriptase by incubation at $42^{\circ} \mathrm{C}$ for $45 \mathrm{~min}$ in $30 \mathrm{mM}$ Tris hydrochloride (pH 8.7) $-5 \mathrm{mM}$ $\mathrm{MgCl}_{2}-2.5 \mathrm{mM}$ dithiothreitol-3.0 mM KCl-350 $\mu \mathrm{M}$ each deoxynucleoside triphosphate. Labeled fragments were analyzed on a $7 \mathrm{M}$ urea-5\% polyacrylamide gel, and the gel was exposed to Kodak XAR-5 X-ray film at $-80^{\circ} \mathrm{C}$ with an intensifying screen.

S1 nuclease assays. S1 nuclease analyses were performed essentially as described previously (39). The BglII-EcoRI fragment of pCHB3-2 was end labeled with the large fragment of DNA polymerase $I$ in the presence of $[\alpha-$ $\left.{ }^{32} \mathrm{P}\right]$ deoxynucleoside triphosphates, while the XhoI-PvuII fragment of pCHB3-2 was end labeled with T4 polynucleotide kinase in the presence of $\left[\gamma^{-32} \mathrm{P}\right] \mathrm{ATP}$. The end-labeled fragments were hybridized with $2 \times 10^{6}$ cell equivalents (reflecting recovery) of total erythroid RNA from 5-, 8-, 10-, and 15-day-old chicken embryos, and the end-labeled BglIIEcoRI fragment was also hybridized to $200 \mathrm{ng}$ of poly(A) ${ }^{+}$ RNA from 5-day-old erythroid cells in $80 \%$ formamide- 40 mM PIPES [piperazine- $N, N^{\prime}$-bis(2-ethanesulfonic acid)] 0.4 $\mathrm{M} \mathrm{NaCl}-1 \mathrm{mM}$ EDTA for $12 \mathrm{~h}$ at $55^{\circ} \mathrm{C}$. The samples were digested with $\mathrm{S} 1$ nuclease (5) for $2 \mathrm{~h}$ at $30^{\circ} \mathrm{C}$, and the fragments were resolved on $7 \mathrm{M}$ urea-5\% polyacrylamide gels. The gels were exposed to Kodak XAR-5 X-ray film at $-80^{\circ} \mathrm{C}$ with an intensifying screen.

Construction of pCHB3-1 $\triangle$ and transcription in vitro. Approximately $5 \mu \mathrm{g}$ of the band $3 \mathrm{cDNA}$ clone, pCHB3-1, was digested with the restriction endonuclease BamHI. The resulting fragments were resolved on a low-melting-point agarose gel, and the 5.7-kilobase fragment was isolated (49). This fragment, which lacks the 716-base-pair (bp) BamHI restriction fragment that extends from the BamHI site in the polylinker to nucleotide -14 relative to the translational initiation site of pCHB3-1, was religated with T4 DNA ligase and used to transform $\mathrm{HB} 101$ cells. Positive colonies were selected, and DNA was prepared. DNA $(5 \mu \mathrm{g})$ from this construct, pCHB3-1 $\Delta$, and $5 \mu \mathrm{g}$ of DNA from pCHB3-1 were linearized with the restriction endonuclease $B g l I$ and used as templates for in vitro transcription reactions with SP6 RNA polymerase (36).

In vitro translations. Approximately $500 \mathrm{ng}$ of RNA transcribed in vitro from both pCHB3-1 and pCHB3-1 $\Delta$ was translated in vitro in a wheat germ extract (Amersham Corp., Arlington Heights, Ill.) in the presence of $0.2 \mathrm{mCi}$ of $\left[{ }^{35} \mathrm{~S}\right]$ methionine $(1,200$ to $1,400 \mathrm{Ci} / \mathrm{mmol}$; Amersham Corp.) per $\mathrm{ml}$ by incubation for $30 \mathrm{~min}$ at $25^{\circ} \mathrm{C}$. The resulting ${ }^{35} \mathrm{~S}$-labeled polypeptides were immunoprecipitated (13) with a previously characterized band 3 antiserum (12). The band 3 immunoprecipitates were analyzed by electrophoresis on a sodium dodecyl sulfate-15\% polyacrylamide gel (33). The gel was impregnated with 2,5-diphenyloxazole, dried, and exposed to Kodak XAR-5 X-ray film at $-80^{\circ} \mathrm{C}$.

RNA isolation. Erythroid cells were isolated from 5-, 8-, 10-, and 15-day-old chicken embryos as described previously (7). The cells were homogenized with a Dounce homogenizer in a solution that contained $5 \mathrm{M}$ guanidinium isothiocyanate, $50 \mathrm{mM}$ Tris hydrochloride (pH 7.5), $50 \mathrm{mM}$ EDTA, 5\% $\beta$-mercaptoethanol, and $3 \%$ sodium lauryl sarcosine. Homogenates were layered over a cushion of $5.7 \mathrm{M} \mathrm{CsCl}$ and centrifuged as described previously (9). Poly(A) ${ }^{+}$RNA was isolated by oligo(dT)-cellulose chromatography (2).

\section{RESULTS}

Primary structure of chicken erythroid anion transporter. A $\lambda$ gt 11 cDNA library constructed with poly(A) ${ }^{+}$RNA from erythroid cells isolated from 15-day-old chicken embryos was screened with a previously described erythroid anion transporter (band 3) cDNA clone, p3(9-1), as a probe (12). Additional cDNA clones were isolated, and one of these clones, pCHB3-1, was shown to contain an insert of 3,448 bp. Subsequent studies revealed that pCHB3-1 encompasses the entire coding region of chicken erythroid band 3 as well as $705 \mathrm{bp}$ of the $5^{\prime}$ untranslated region and $177 \mathrm{bp}$ of the $3^{\prime}$ untranslated region. Primer extension analysis indicated that the band 3 mRNA extends $\sim 850$ bases $5^{\prime}$ of pCHB3-1, consistent with a size of 4.3 kilobases for the RNA (12). The nucleotide sequence of pCHB3-1 is presented in Fig. 1. A single open reading frame extends from nucleotide 1 to nucleotide 2532 and encodes a predicted polypeptide of 844 amino acids, which is significantly shorter than the 929 amino acids predicted for murine erythroid band 3 (30). 
-705 AGT ATC CCC ATC ATC CAC TTT GTT GTC TM AM AM CCC AM ACC TAC AM ACA CAC ACA ACC ACT CTT CTA CAC CTA CTC GCG TCG GCG TAT CTT CTC TGA CCA ATC ATC ACA CCT GTA -585 CCA TAT AGA ANG ANG TA TAT TEG ACA MM TCC TEG TAC AGA AGT CTC MAT GTC ITA ATC ATC TCC ACT AM GCC TCT GTC GTA CTA CTC ATT CCA GAC CCT GGT ATT ACA TCA AMT GCC -465 ACA CCA TGT GTA CCA AM TCA CGT GTC ATG TAC TAC ACG ATT TAT TCA TTA GGC TCA ACT ATT TTC TAT TTA CM AGT TCG TCC TTC TTT GAT CAG GGT GAT AGA ATT TCT CAC TCC TA

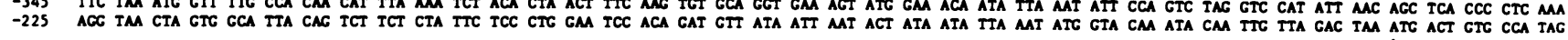

-105 AMC CM ATC CAM ANC ACC TCT CAG GTT GM TCT GM TAT TAT GTA GCT CTA GCC GAC ACG AAC CTC CAC GAC TTC GTC CTC GAC ACC ACC MAC GAT CCC TCC TCC ATC ATC GAC GCC GCC CCC 16 TCG CTC CAT CTC GAS GAC ACC ATG GAC CCG GGG GCG GCG TCG GCC ACC CAC CTC CCC CTC CTC ACC TAC CAC ACC CTC CTC GAC CTC CAC CGC GCC TTC GCC A0 MAM GGC GTT GTC CTC CTC

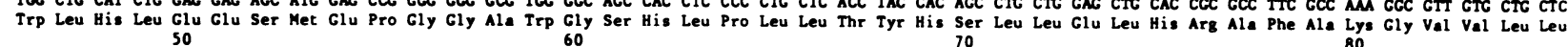
136 GAC GTC GCC GCC ACC TCC CTC GCA CCC CTC GCC CAC GTC CTG CTC GAT CAC CTC ATC TAC GAC GCG CAC CTC 70 AAC CCC CAC CAC CGC GAC GAC GTC CTC CCG GCC CTC CTC CTC CGC CAC

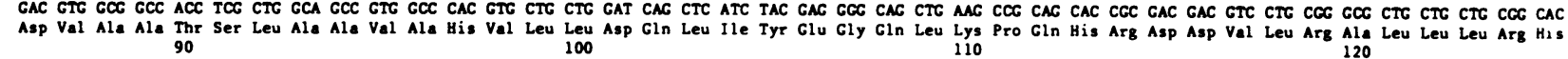

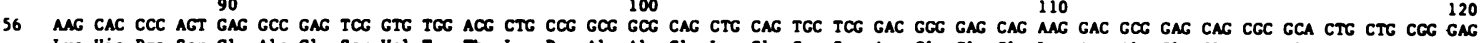

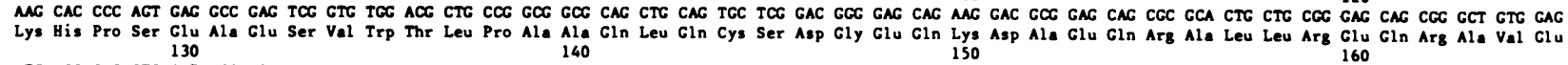
376 ATC AGG GAC CTC CAT GGG GCC GGC CAG AGC CCC TCC AGC GCC CAC CTC GGC CCA CAC CTC CAC CAC CAC CTC CCC GAC GAC ACC GAC GCC ACC CTC GTC CTC GTC GCC TCC GCA GCC TTC Met Arg Glu Leu His Gly Ala Gly Gln Ser Pro Ser Arg Ala Gln Leu Gly Pro Gln Leu His Gin Gln Leu Pro Glu Asp thr Glu Ala thr Leu Val Luu Val Ala Cys Ala Ala Phe

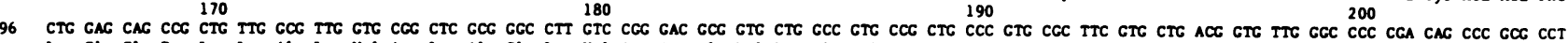

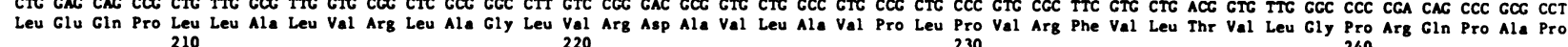
16 CAG CTA CCA CGA GAT CGC CGC GCC GCC GCC ACC GTC ATC GCC GAC CGC GTC TTC CGC CGC GAC GCC TAC CTC TGC GGC GGC CGT GCC GAC CTC CTG GGC GGC CTC CAC GGC TTC CTC GAC

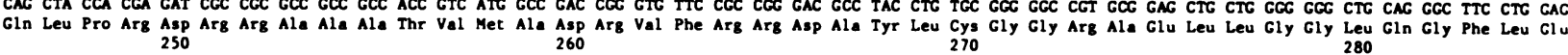

136 GCC AGC ATC GTT CTC CCG CCC CAM GAC GTG CCC AGC GAG CAC CAC CTG CAT GCC CTC ATC CCA CTC CAC CGC CAC GCT GTC CGC CGC CGC TAC CAC CAC CCC GAC ACC GTC CGC ACC CCC

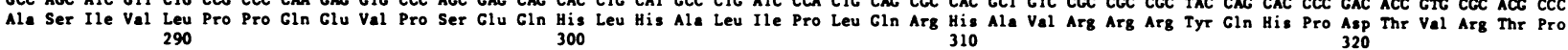
856 GGC GGC CCC ACG GCC CCC AM GAC ACA GGC GAT AAC GGC CAC GCT CCC CAC GAC GAC GAC CCC CTC CTC COC ACC AGC COC CCC TTT GCC GGC TTC CTC ACC GAC ATC CGC CGC CGT TAC

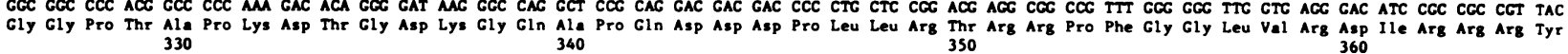

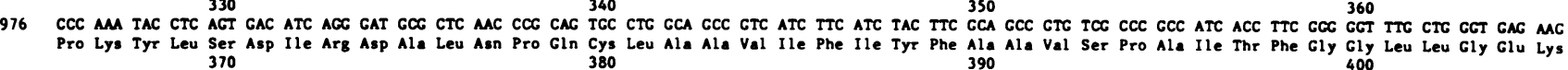
096 ACC CCC GGT ATC ATC GCC GTC TCC GAC CTC CTC CTC TCC ACC 380 AGC GTC CAC TCT TTC CTC TTC AGT CTC CTC 390 AGC GCC CAC CCT CTC CTC GTC GTC GCC TTC TCC GCC CCA CTC CTC GTC

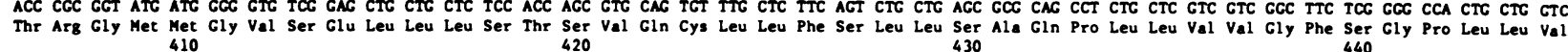
216 TTT GAC GAC CCT TTC TTC ACC TTC TCT GAC GAT CAT GCC CTC 420 GAC TAC ATC CTC CCC CGC CTC TCC ATC GCC 430 TTC TCC CTC ATC CTC CTC GTC CTC CTC GTC GTC GCC TCC GAC GGC ACC

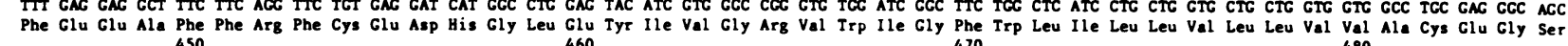

1336 CTC CTC GTC CCC TAC CTC TCC CCA TAC ACC CAC CAC ATC TTC TCC TTC CTC ATC TCC CTC ATC TTC ATC TAT GAC ACC TTC GCC AM CTC CTC ACC ATC TTC CAC GCC CAC CCC CTC CAC

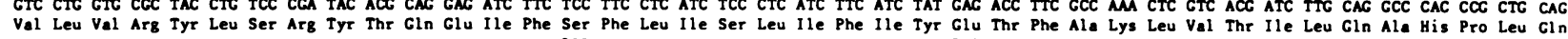

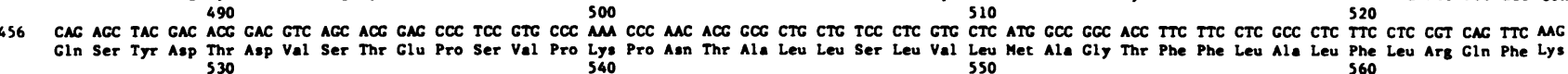

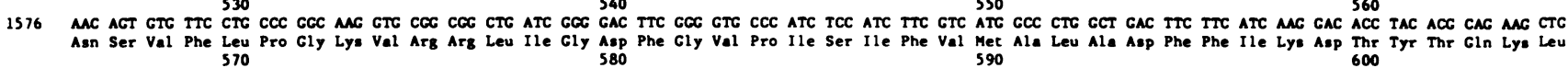

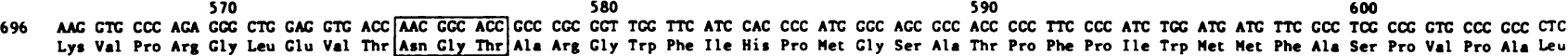

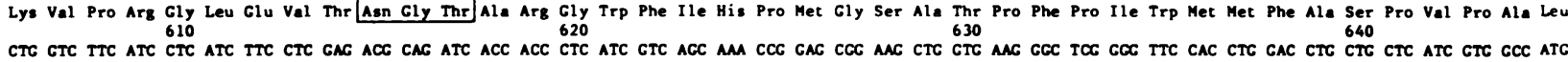

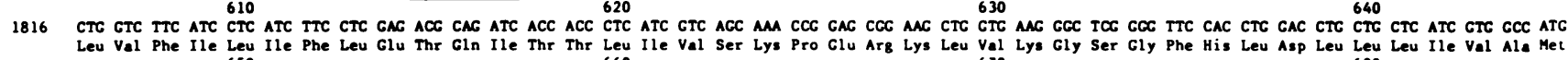
936 GGC GCC CTC GCC GCC CTC TTC GCC ATC CCC TCG CTC ACC GCC 660 ACC ACC CTC CCC ACC ATC ACC CAC GCC AAC GCC CTC ACC GTC GTC GGT AAC AGC GCC GTC COC GCC GAC ACC GCC CAC

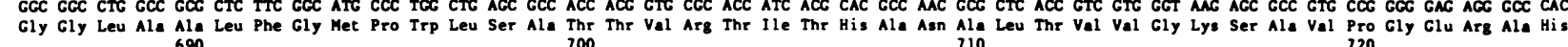
2056 ATC GTC GAC GTC AAC GAC CAC CGC CTC AGC GGC CTC CTC GTC GCC GTC CTC ATC GGC GTC TCC ATC CTC ATC GAC CCC ATC CTC AAC TAC ATC CCC CTC GCC CTC CTC TTC GGC ATC TTC

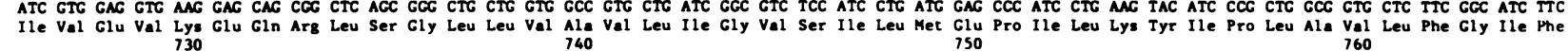

2176 CTC TAC ATC GGC GTC ACC TCC CTC TTC CCC ATC CAC CTC TTC GAC CGC ATT CTC CTC CTC CTC ATC CCC CCC 750 TAC CAC CCC AAC GAC CCC TAC GTC ACC CGC CTC AAC ACC TCC CCC

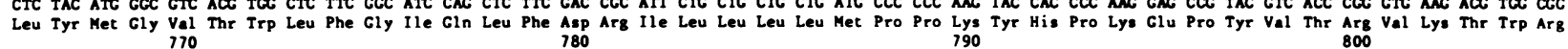

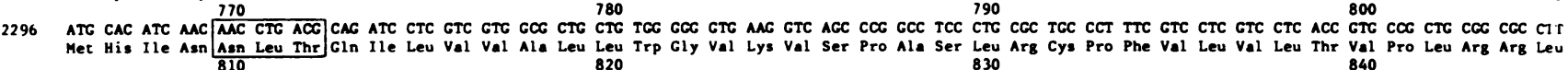

2416 CTC CTG CCC CGC ATC TTC AGC GAC ATC GAC CTC AMA TCC CTG GAC ACC GAC GAC GCA GTC GTC ACA TTT GAM GAC GCC GAC GGC CAC GAC GTC TAC AAC GAC GTC CAC ATC CCC AGC TAA

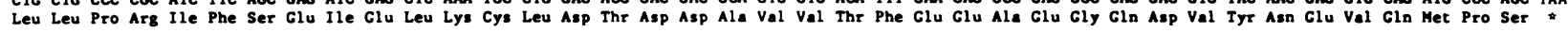

2536 GGT CCC GCC GTC CCC CCA CCC ACG TGT AGA TCC ACC AGT CGC CCC CCC AAC ACC CGC GTC CTC CCC AGA GCC TCC CGC TAT GCC GCC CAC CGT CCCC GCC GTA GCC ATC GM CAC CCC ACC 2656 ACA GGG GCT ACC GGT TTC TM CGC AGA GM TCG CTC GCA MM ACA CCA AM AM AMA AMA AM AM AM

FIG. 1. Nucleotide sequence and predicted amino acid sequence of the chicken band 3 mRNA. The nucleotide sequence of pCHB3-1 is shown with the nucleotide numbers in the left-hand margin. The probable translational initiation site is designated by the ATG codon at nucleotide 1 . Amino acids are numbered above each line. The boxes indicate the potential glycosylation sites at nucleotides 1723 and 2308 .

The majority of this difference resides in the N-terminal region of the molecule where an additional 89 amino acids are predicted in mouse band 3 that are absent in chicken band 3 (Fig. 2). Two lines of evidence suggest that translational initiation of chicken erythroid band 3 occurs at the ATG at nucleotide 1 (Fig. 1). First the Met codon beginning at nucleotide 1 is the first in-frame ATG downstream from the stop codon (TAG) at nucleotide -108 . Second, translation in a wheat germ extract of in vitro RNA transcripts that extend from the 5' end of pCHB3-1 to the first $B$ glI site (Fig. $3 \mathrm{~A}$ ) resulted in a single polypeptide of $\sim 32,000$ daltons that was immunoprecipitable with chicken erythroid band 3 antibodies (Fig. 3B, lane 2). This result is in agreement with the predicted mass of 33,000 daltons for a polypeptide that initiates at nucleotide 1 and terminates at the $B g l I$ site. To ensure that this translation product did not initiate at an ATG upstream of nucleotide 1 and result from premature transla- tional termination or degradation, we deleted the region from nucleotides -705 to -14 of pCHB3-1 by excision of a 716-bp BamHI fragment (Fig. 3A). Transcripts made in vitro from this construct, $\mathrm{pCHB} 3-1 \Delta$, which extended from nucleotide -13 to the $B g I I$ site were translated, and the resultant polypeptides were immunoprecipitated with chicken erythroid band 3 antibodies. These truncated transcripts also gave rise to a polypeptide of $\sim 32,000$ daltons, (Fig. 3B, lane 3 ), which provides further evidence that initiation occurs at nucleotide 1 . The minor translation products of $\sim 31,000$ daltons that were detected in the immunoprecipitates in Fig. $3 \mathrm{~B}$, lanes 2 and 3, suggested that translational initiation in vitro also occurs at Met residue 13 (Fig. 1), whose surrounding sequences possess greater homology to the consensus sequence flanking functional eucaryotic initiation sites (31) than those surrounding Met residue 1 . The predicted size of 109,000 daltons for the chicken band 3 polypeptide that 


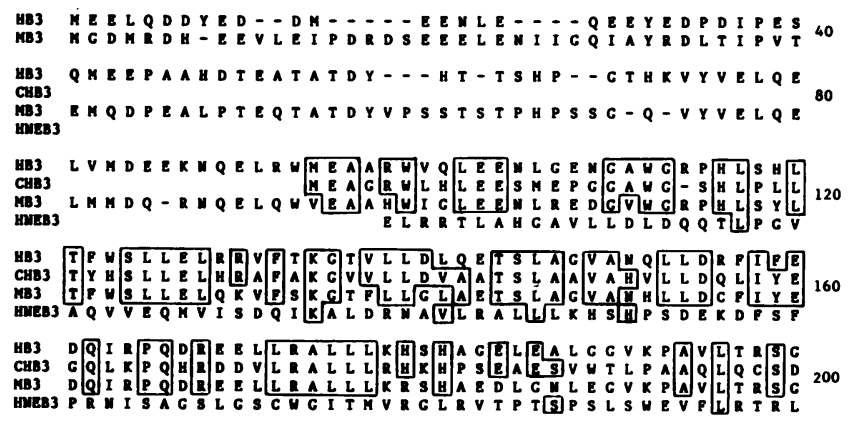

HB3 DPSOPLLPQASSLRT $Q$ LP

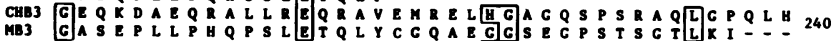
$M a 3$
HMEB

CHB3 Mas

c883

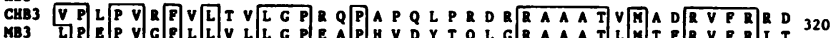

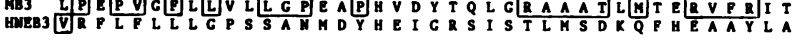

нв3

CaB3

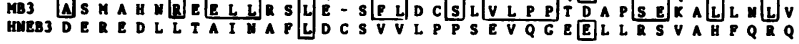
HB3

CHB3 $M B 3$
MUEB

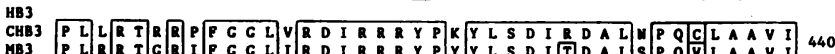

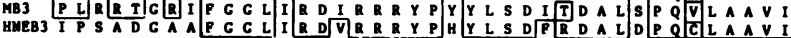

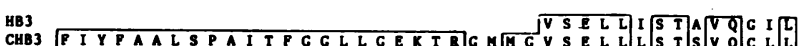
CHBB3

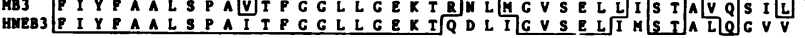
Hв3 PA

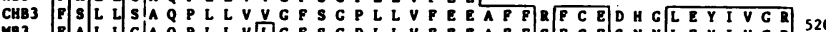

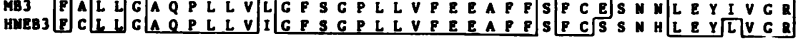

年3 Bues3

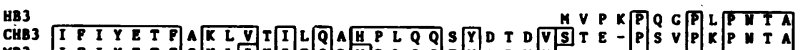

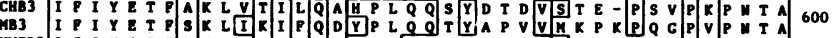

maEs

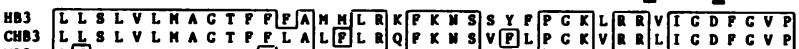

CHE3

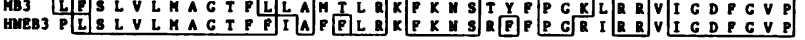
HB3

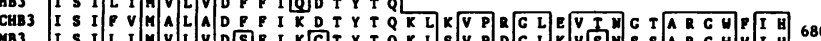

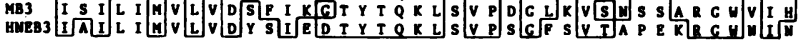

CHB3

CAB3 MAB

CHB3 PERK

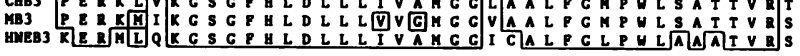

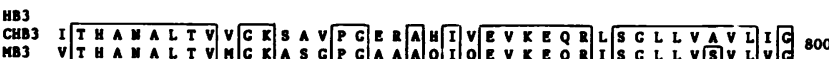

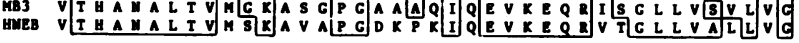

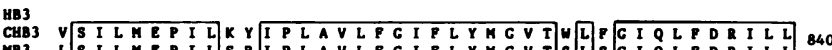

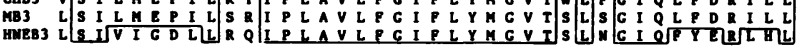

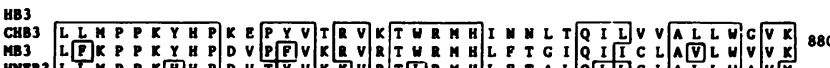

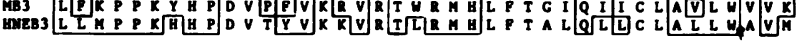

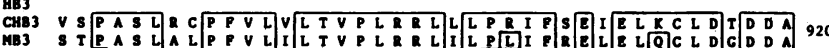

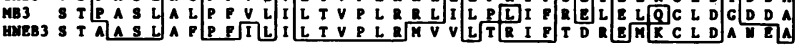

MBs3

Mas

960

FIG. 2. Comparison of the amino acid sequence of the chicken erythroid band 3 polypeptides with those of the murine and human erythroid and the human nonerythroid band 3 polypeptides. Amino acid sequences were aligned to optimize homology, except for the initiates at nucleotide 1 agrees well with the estimates obtained from sodium dodecyl sulfate-polyacrylamide gels of 100,000 to 105,000 daltons for this protein $(12,22)$.

The hydropathy plot in Fig. 4 indicates that chicken erythroid band 3 can be divided into a hydrophilic $\mathrm{N}$ terminal cytoplasmic domain and a hydrophobic C-terminal transmembrane domain. Previous studies have shown similar results for the human $(17,20,45,46)$ and murine (30) erythroid as well as the human nonerythroid (15) band 3 polypeptides. The truncated cytoplasmic domain of chicken erythroid band 3 is consistent with the observation that chicken band 3 does not bind glyceraldehyde-3-phosphate dehydrogenase (22), which interacts with the amino terminus of human erythroid band 3 (47). The absence of this highly acidic N-terminal region (Fig. 2) from the chicken band 3 polypeptide also correlates with the isoelectric point of chicken band 3, which is 1 to $2 \mathrm{pH}$ units more basic than that of human band 3 (22). In agreement with this result, sequence analysis reveals a net positive charge ( 54 basic and 40 acidic residues) for the cytoplasmic domain of chicken band 3 , which extends approximately to residue 435 in Fig. 2, while the cytoplasmic domain of murine band 3 exhibits a net negative charge (65 acidic and 37 basic residues) (30). This observed charge difference may result in conformational changes in the cytoplasmic domain of chicken band 3 that render it much more resistant to proteolysis with trypsin than human band 3 (22).

Electron microscopic (48) and hydrodynamic (1) studies of the cytoplasmic domain of human band 3 suggest that this region of the molecule forms an elongated homodimer with a $\mathrm{pH}$-regulated hinge region that is rich in proline residues. The chicken band 3 polypeptide initiates in a tryptophan-rich region (1) just before this proline-rich region which is between residues 165 to 208 (Fig. 2). The ankyrin-binding site of band 3 has been tentatively placed between this tryptophan-rich region and the $\mathrm{pH}$-regulated hinge of the cytoplasmic domain (34). It is interesting to note that the overall similarity between the cytoplasmic domains of chicken band 3 and murine band 3 is only $43 \%$, while the similarity of this putative ankyrin-binding site (between residues 124 and 164; Fig. 2) is approximately $70 \%$ between the chicken band 3 and the murine and human erythroid band 3 polypeptides. These results suggest that although the sequence of the cytoplasmic domain has diverged considerably, the sequence of the high-affinity ankyrin-binding site has been relatively conserved among different species.

The hydropathy plot of the chicken band 3 polypeptide reveals 10 very hydrophobic regions (A through $J$ in Fig. 4) in the C-terminal portion of the molecule that are similar to those previously demonstrated for the murine erythroid (30) and human nonerythroid (15) band 3 polypeptides. These 10 hydrophobic regions within the transmembrane domain correspond to the putative membrane-spanning segments of the

$\mathrm{N}$-terminal region of the human nonerythroid band 3 polypeptide. The numbers of the amino acids (including the inserted dashes) are indicated in the margin to the right. The boxed regions indicate identity between the chicken erythroid band 3 polypeptide and the erythroid and nonerythroid band 3 polypeptides from other species. The arrowheads after residues 592 and 596 indicate insertions in the transmembrane domain of the human nonerythroid band 3 polypeptide of $\mathbf{2 5}$ and $\mathbf{3}$ amino acids, respectively, that are not included in the figure. The $\mathrm{B}$ at residue 389 of human erythroid band 3 corresponds to Asn or Asp. CHB3, Chicken erythroid band 3; MB3, murine erythroid band 3; HB3, human erythroid band 3; and HNEB3, human nonerythroid band 3 . 


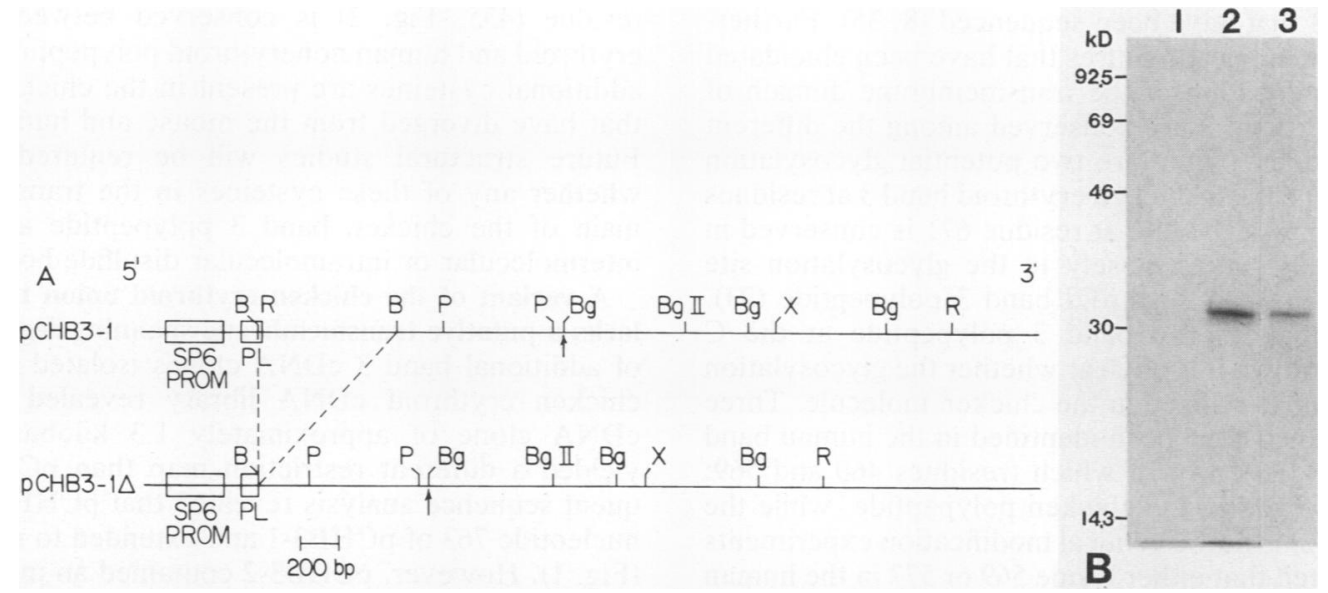

FIG. 3. In vitro translation and immunoprecipitation with band 3 antibodies of truncated band 3 polypeptides. Transcripts were synthesized in vitro with SP6 RNA polymerase from pCHB3-1 and pCHB3-1 $\Delta$ templates (prepared as described in Materials and Methods) that were linearized with restriction endonuclease BglI (A). These transcripts, which terminate at the BglI site indicated by the arrows (A), were translated in vitro in a wheat germ extract in the presence of $0.2 \mathrm{mCi}$ of $\left.{ }^{35} \mathrm{~S}\right]$ methionine per ml, and the resulting polypeptides were immunoprecipitated with band 3 antibodies (B). The band 3 immunoprecipitates resulting from the translation of pCHB3-1 transcripts (lane 2), pCHB3-1 $\Delta$ transcripts (lane 3), and a no RNA control (lane 1) were analyzed by electrophoresis on a sodium dodecyl sulfate-15\% polyacrylamide gel. The migration of ${ }^{14} \mathrm{C}$-labeled molecular mass markers (phosphorylase $b, 92,500$ daltons ( $92.5 \mathrm{kD}$ ); bovine serum albumin, 69,000 daltons; ovalbumin, 46,000 daltons; carbonic anhydrase, 30,000 daltons; and lysozyme, 14,300 daltons) is indicated in the margin to the left. Abbreviations in the restriction maps are: PL, polylinker; B, BamHI; R, EcoRI; P, PvuII, Bg, BglI; BgII, BglII; X, XhoI; PROM, promoter.

polypeptide. Hydrophobic peaks A, C, E, and G (Fig. 4) are composed primarily of hydrophobic amino acids and fit the criteria for single membrane-spanning $\alpha$-helical structures (18). Hydrophobic peaks D and F (Fig. 4) contain both hydrophilic and hydrophobic amino acids and are predicted to form single membrane-spanning amphipathic helices with all the polar residues on one face of the helix. Hydrophobic peak B is similarly composed of hydrophilic and hydrophobic residues and is predicted to form an amphipathic helix which spans the membrane two times, consistent with the model proposed for murine erythroid band 3 (30). Recent experiments with monoclonal antibodies have demonstrated that lysine 772 (Fig. 2) is susceptible to intracellular cleavage with trypsin (25). This result necessitates that hydrophobic peak H (Fig. 4) spans the membrane two times or not at all to maintain the proper orientation of the band 3 polypeptide in the membrane. The highly hydrophobic nature of peak $\mathrm{H}$ suggests that this region indeed traverses the membrane two times in an $\alpha$-helical structure which turns within the membrane at proline 751 (Fig. 2). Since no topological data are available for the extreme $C$ terminus of the band 3 polypeptide it is not possible at present to predict the number of times hydrophobic regions I and J (Fig. 4) cross the membrane.

The similarity observed in the hydropathy plots of the band 3 polypeptides from different species is consistent with the high degree of conservation at the sequence level. The transmembrane domain of the chicken band 3 polypeptide is $79 \%$ similar to the murine erythroid and $71 \%$ similar to the human nonerythroid band 3 polypeptides (residue 435 to $\mathrm{C}$ terminus; Fig. 2). Chicken band 3 also exhibits $75 \%$ similarity to those regions of the transmembrane domain of human

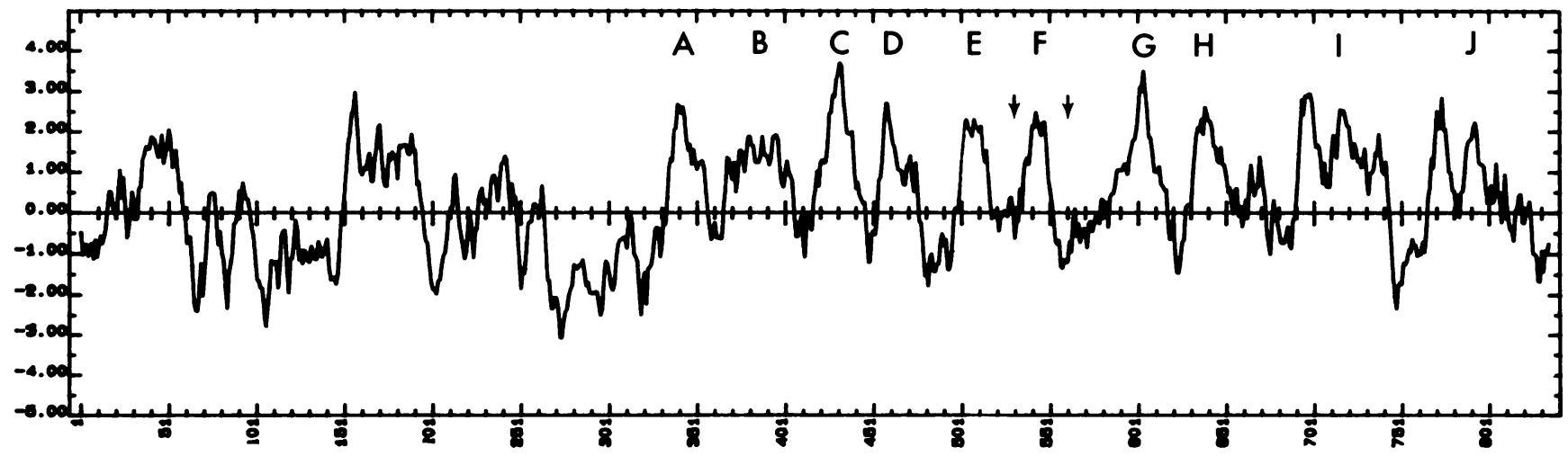

FIG. 4. Hydropathy plot of the predicted amino acid sequence of the chicken erythroid band 3 polypeptide obtained from the nucleotide sequence of pCHB3-1. The plots were made by using the algorithm of Kyte and Doolittle (32). Hydrophobicity values were averaged over a window of seven amino acids. Hydrophobic regions in the sequence are designated A to J. The arrowheads at residues 334 and 358 indicate the boundaries of the region that is present in pCHB3-1 and absent in pCHB3-2. Note that this region almost exactly corresponds to hydrophobic region $\mathrm{F}$. 
erythroid band 3 that have been sequenced $(8,35)$. Furthermore, various topological features that have been elucidated by chemical modification of the transmembrane domain of human erythroid band 3 are conserved among the different band 3 polypeptides. There are two potential glycosylation sites (Asn-X-Ser/Thr) in chicken erythroid band 3 at residues 671 and 866 (Fig. 2). The site at residue 671 is conserved in murine band 3 and maps closely to the glycosylation site identified in the human erythroid band 3 polypeptide (23). Since the topology of the band 3 polypeptide at the $C$ terminus is not known it is unclear whether the glycosylation site at residue 866 is utilized in the chicken molecule. Three extracellular lysines have been identified in the human band 3 polypeptide $(8,35)$, two of which (residues 460 and 569 ; Fig. 2) are conserved in the chicken polypeptide, while the third (residue 572) is not. Chemical modification experiments have demonstrated that either lysine 569 or 572 in the human erythroid polypeptide is modified by di-iodosulphophenyl isothiocyanate $\left(\mathrm{H}_{2} \mathrm{DIDS}\right)$, an inhibitor of anion transport (35). $\mathrm{H}_{2}$ DIDS-sensitive sites have been suggested to be high-affinity chloride-binding sites in human band 3 . These results suggest that lysine residue 569 provides one of the high-affinity chloride-binding sites in the chicken band 3 polypeptide.

A 72-amino-acid peptide fragment from human erythroid band 3 has been shown to contain an internal lysine (residue 619; Fig. 2) that is modified by phenylisothiocyanate (PITC), which blocks anion transport (8). This lysine is not conserved in the chicken erythroid band 3 polypeptide. This suggests that the PITC modification of lysine residue 619 blocks anion transport by steric inhibition rather than interacting with an essential residue for the transport process. Tyrosine residue 657 (Fig. 2) in this peptide fragment is susceptible to extracellular radioiodination (8) and is conserved among the band 3 polypeptides from different species. Finally, modification of carboxylic groups in this region of the polypeptide inactivates anion exchange (6). Three aspartate residues $(636,650$, and 655 ; Fig. 2) are conserved among the chicken and human erythroid and human nonerythroid band 3 polypeptides, although aspartate 655 has diverged in the murine sequence. This result suggests that aspartate residue 636 or 650 is important in the exchange process.

It is interesting to note that nine of the membranespanning segments of the chicken band 3 polypeptide are bound internally or externally by arginine residues and that each of these residues is conserved in either the murine erythroid or human nonerythroid polypeptide. Chemical modification $(51,53)$ and $\mathrm{pH}$ titration (50) experiments have indicated that arginine residues in the 35,000-dalton chymotryptic fragment of the membrane domain of human erythroid band 3 are involved in anion exchange. The location of these arginine residues in the membrane-spanning segments of the chicken band 3 polypeptide suggests that one or more of these residues provides additional high-affinity $\mathrm{Cl}^{-}$. binding sites for the chicken band 3 polypeptide.

Human erythroid band 3 has been shown to exist as a dimer in solution in the presence of nonionic detergents (52). This interaction is mediated by the transmembrane domain since the removal of the cytoplasmic domain by proteolysis does not affect dimerization (41). This dimerization of the transmembrane domain could presumably be mediated by disulfide linkages. Sequence comparison reveals that two cysteine residues (509 and 914 ; Fig. 2 ) in the transmembrane domain are conserved among the chicken and murine erythroid and human nonerythroid band 3 polypeptides. A third residue (435; Fig. 2) is conserved between the chicken erythroid and human nonerythroid polypeptides, while three additional cysteines are present in the chicken polypeptide that have diverged from the mouse and human sequences. Future structural studies will be required to determine whether any of these cysteines in the transmembrane domain of the chicken band 3 polypeptide are involved in intermolecular or intramolecular disulfide bonding.

$A$ variant of the chicken erythroid anion transporter that lacks a putative transmembrane-spanning segment. Analysis of additional band $3 \mathrm{cDNA}$ clones isolated from the $\lambda \mathrm{gt} 11$ chicken erythroid cDNA library revealed that a partial cDNA clone of approximately 1.3 kilobases, pCHB3-2, yielded a different restriction map than pCHB3-1. Subsequent sequence analysis revealed that pCHB3-2 initiated at nucleotide 763 of pCHB3-1 and extended to nucleotide 2030 (Fig. 1). However, pCHB3-2 contained an in-frame deletion of 75 nucleotides in the transmembrane domain of the chicken band 3 polypeptide. This deletion encompassed nucleotides 1601 to 1675 that encode amino acids 534 to 558 in pCHB3-1 (Fig. 1), which corresponds to residues 630 to 654 in Fig. 2. The arrows on the hydropathy plot in Fig. 4 indicate that the deletion in pCHB3-2 almost exactly corresponds to the membrane-spanning segment represented by hydrophobic peak F. To ensure that pCHB3-2 is represented in the erythroid RNA population rather than simply resulting from an artifact of cloning, we performed $\mathrm{S} 1$ nuclease analysis to examine the $5^{\prime}$ and $3^{\prime}$ boundaries of the deletion. Total erythroid RNA from erythroid cells isolated from 5-, 8-, 10-, and 15-day-old chicken embryos was hybridized to probes end labeled with ${ }^{32} \mathrm{P}$ at the BglII site (Fig. 5A) and the XhoI site (Fig. 5B) as indicated in Fig. 5C. S1 nuclease digestion of the resulting hybrids yielded full-length protected fragments of 656 bp (Fig. 5A) and 1,070 bp (Fig. 5B), representing the RNA species complementary to pCHB3-2, as well as the 225-bp fragment (Fig. 5A) and the 164-bp fragment (Fig. 5B) that would be predicted from hybridization to the RNA species complementary to pCHB3-1. The origin of the additional bands in Fig. $5 \mathrm{~B}$ is at this time unknown. The RNA complementary to pCHB3-2 accumulated during embryonic development in a fashion similar to that previously reported for the RNA complementary to pCHB3-1 (13). Furthermore, analysis of poly $(A)^{+}$RNA from 5-day-old erythroid cells by similar methods (Fig. 5A) indicated that the transcripts complementary to pCHB3-1 and pCHB3-2 are represented in poly(A) ${ }^{+}$RNA to the same extent as they are in total erythroid RNA. These results indicate that RNA transcripts that encode band 3 polypeptides with alternative primary structures in their transmembrane domain are present in chicken erythroid cells.

\section{DISCUSSION}

In this report, we described the cloning and characterization of the chicken erythroid anion transporter (band 3). Analysis of the band 3 cDNA clones revealed several features of chicken erythroid band 3 that are unique among previously characterized band 3 polypeptides, as well as the highly conserved nature of the transmembrane domain of this polypeptide among different species. Furthermore, we described for the first time the characterization of a band 3 polypeptide with alternative primary structures in its membrane-spanning segments.

Sequencing studies in conjunction with primer extension analyses indicated that the chicken erythroid band 3 mRNA contains a very long $5^{\prime}$ untranslated region of $\sim 1.5$ kilobases 


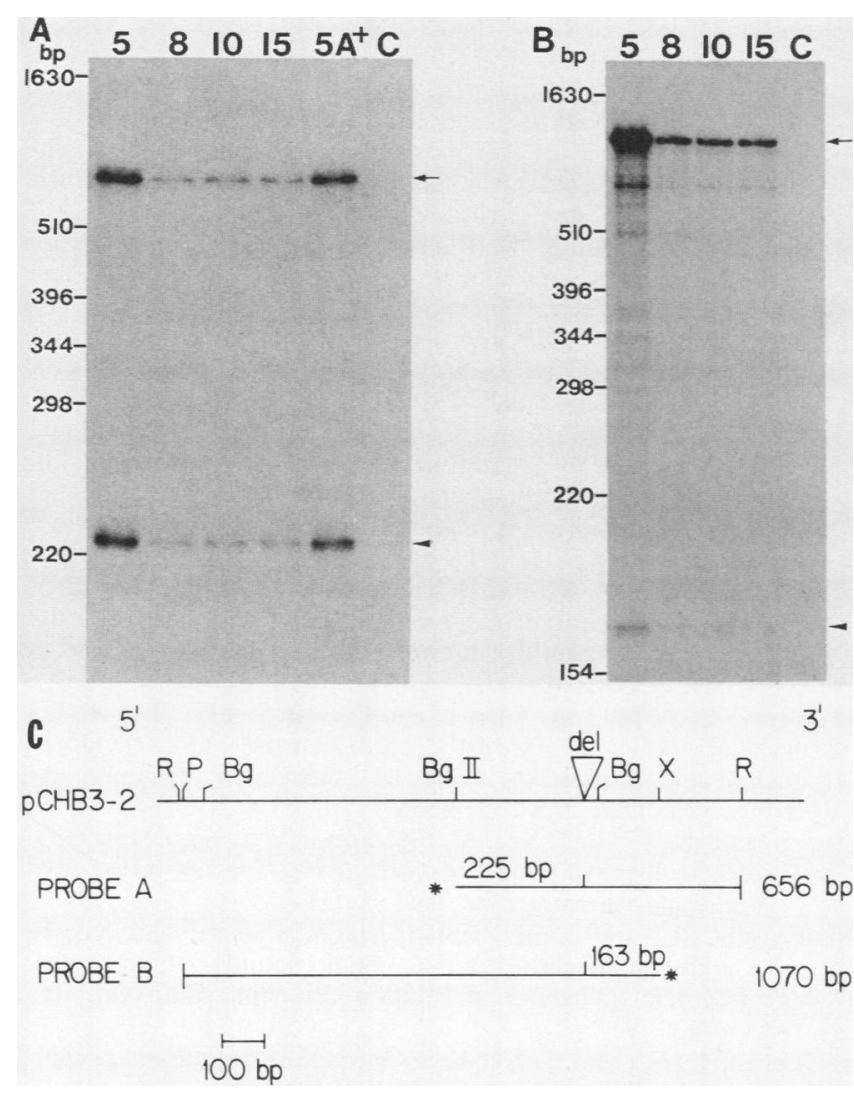

FIG. 5. Steady-state levels of erythroid band 3 RNA determined by $\mathrm{S} 1$ nuclease protection assays. A total of $2 \times 10^{6}$ cell equivalents of total RNA from erythroid cells isolated from 5-, 8-, 10-, and 15-day-old chicken embryos were hybridized with either a ${ }^{32} \mathrm{P}$-endlabeled $B g$ III-EcoRI fragment (A) or a ${ }^{32} \mathrm{P}$-end-labeled XhoI-PvuII fragment (B) from pCHB3-2 for $12 \mathrm{~h}$ at $55^{\circ} \mathrm{C}$. The ${ }^{32} \mathrm{P}$-end-labeled $B g$ III-EcoRI fragment was also hybridized to $200 \mathrm{ng}$ of poly $(\mathrm{A})^{+}$ RNA from 5-day-old erythroid cells for $12 \mathrm{~h}$ at $55^{\circ} \mathrm{C}$ (A). The samples were digested with $\mathrm{S} 1$ nuclease for $30 \mathrm{~min}$ at $30^{\circ} \mathrm{C}$, and the fragments were resolved on a $7 \mathrm{M}$ urea-5\% polyacrylamide gel. Lane C, No RNA control. The protected fragments from each hybridization correspond to those predicted from hybridization of probes A and B to RNA species homologous to pCHB3-1 (225 and $164 \mathrm{bp}$, respectively, marked by arrowheads in panels A and B) and pCHB3-2 (656 and 1,070 bp, respectively, marked by arrows in panels A and B) as illustrated in panel C. Size markers in base pairs are several end-labeled restriction fragments of Hinfl-digested pBR322 DNA. Abbreviations in the restriction map are: R, EcoRI; P, PvuII; Bg, BglI; BgII, BglII; and X, XhoI.

and a $3^{\prime}$ untranslated region of only $177 \mathrm{bp}$. Although there is no consensus polyadenylation signal in the $3^{\prime}$ untranslated region of pCHB3-1 (Fig. 1), multiple cDNA clones have been isolated and sequenced and all terminated at the same poly(A) tract, suggesting that this is the true $3^{\prime}$ terminus of the molecule. The structure of the chicken erythroid band 3 mRNA is different from that observed for the murine band 3 mRNA species, which has been shown to contain a 121-bp $5^{\prime}$ untranslated region and a 1,470-bp $3^{\prime}$ untranslated region (30). Whether this difference in RNA structure seen between the chicken and murine erythroid band 3 RNAs affects factors such as RNA processing, transport, or stability during erythroid development awaits further analysis.

The $\mathrm{N}$ terminus of the chicken erythroid band 3 polypeptide is $\sim 90$ amino acids shorter than that of the murine (30) and the human (26) erythroid band 3 polypeptides. This truncated chicken band 3 polypeptide is consistent with the absence of binding to glyceraldehyde-3-phosphate dehydrogenase (22), which associates with the extreme $\mathbf{N}$ terminus of the human erythroid band 3 polypeptide (47). In addition, the loss of this very acidic $\mathrm{N}$-terminal region accounts for the relatively basic isoelectric point observed for chicken erythroid band 3 (22). Comparison of the sequence of the cytoplasmic domain of chicken and murine erythroid band 3 polypeptides indicates that the degree of conservation is relatively low. However, the region where the high-affinity ankyrin-binding site has tentatively been placed (34) exhibits greater than $70 \%$ identity between the chicken erythroid and the murine and human erythroid band 3 polypeptides. In addition, regions of the human nonerythroid band 3 polypeptide (residues 98 to 121 and 142 to 153; Fig. 2) also possess considerable similarity to this putative ankyrin-binding site, suggesting that this site is conserved not only in erythroid but also in nonerythroid cell types.

The transmembrane domain of chicken erythroid band 3 is highly homologous to the transmembrane domains of the murine erythroid and human nonerythroid band 3 polypeptides. Each of these polypeptides possesses 10 hydrophobic regions which potentially span the membrane 12 to 14 times. Four of these regions (peaks A, C, E, and G; Fig. 4) are predicted to span the membrane a single time in an $\alpha$-helical structure, consistent with the model for murine erythroid band 3 (30). The recent demonstration that lysine 772 (Fig. 2) is intracellular (25) requires that hydrophobic peak $\mathrm{H}$ (Fig. 4) spans the membrane twice or not at all to maintain the proper topology of the band 3 polypeptide in the lipid bilayer. The highly hydrophobic nature of peak $H$ suggests that it traverses the membrane two times, unlike the model for murine band 3 which proposes a single membranespanning segment (30). Two of the hydrophobic regions (D and F; Fig. 4) are predicted to cross the membrane once as amphipathic helices, whereas region $B$ crosses the membrane two times as an amphipathic helix. Since it is not known whether the $C$ terminus of the band 3 polypeptide is internal or external it is not possible to predict with certainty the number of times hydrophobic peaks $I$ and $J$ cross the membrane (Fig. 4). However, given that both peaks are very broad ( 20 to 24 amino acids per membrane-spanning segment), it is possible that each traverses the membrane two times as an amphipathic helix, resulting in 14 membranespanning segments with the $C$ terminus being intracellular. Additional biochemical and structural studies will be required to assess the validity of this model for the topology of the band 3 polypeptide.

The demonstration of RNA transcripts in chicken erythroid cells that encode variant band 3 polypeptides provides the first example of a transporter protein or an ion channel that exhibits alternative primary structures in its membranespanning segments. DNA sequencing and $\mathrm{S} 1$ analyses showed that the RNA complementary to pCHB3-2 lacks amino acids 534 to 558 of pCHB3-1, which corresponds almost exactly to hydrophobic peak $F$ of the hydropathy plot. Recent studies have demonstrated that the chicken erythroid band 3 polypeptide is composed of at least two variants of $\sim 100,000$ and $\sim 105,000$ daltons, both of which label with $\mathrm{H}_{2}$ DIDS $(12,24)$. Pulse-chase studies have further shown that these variant band 3 polypeptides arise through the posttranslational modification of $\sim 95,000$ - and $\sim 97,000$ dalton primary translation products (13). The 25 -amino-acid segment that is absent in the polypeptide corresponding to pCHB3-2 suggests the possibility that $\mathrm{pCHB} 3-2$ corresponds to the $\sim 95,000$-dalton translation product. Examination of 
the structure and organization of the murine band 3 gene (29) has revealed that one of the intron-exon splice junctions of this gene exactly matches the $5^{\prime}$ boundary of the deletion in pCHB3-2. The $3^{\prime}$ boundary of the deletion in pCHB3-2 is 14 nucleotides $5^{\prime}$ to the next intron-exon splice junction in the murine band 3 gene. This strongly suggests that the equivalent of murine exon 15 in the chicken gene is removed from the RNA corresponding to pCHB3-2 by alternative splicing. This hypothesis is further supported by the fact that the murine (29) and probably the chicken (12) band 3 polypeptides are encoded by a single gene. The human nonerythroid band 3 polypeptide contains two hydrophilic insertions (arrowheads, Fig. 2) of 25 and 3 amino acids that are probably extracellular and are not found in the chicken or murine erythroid band 3 polypeptides. These two insertions may also result from alternative splicing of the human nonerythroid band 3 RNA.

At present, it is not known whether the polypeptide encoded by pCHB3-2 stably accumulates in the membrane of chicken erythroid cells, although the transcript complementary to pCHB3-2 accumulates in the same manner as that complementary to pCHB3-1 during chicken embryonic development. The levels of the two RNA species detected by $\mathrm{S} 1$ nuclease assays (Fig. 5) do not reflect their relative abundance. Additional experiments have indicated that the RNA complementary to pCHB3-1 is much more abundant than the RNA complementary to pCHB3-2 at all stages of development examined (data not shown).

The stable accumulation of the polypeptide encoded by pCHB3-2 in the erythrocyte membrane would not necessarily indicate that this polypeptide could act as a functional anion transporter. A current anion transport model has proposed that the helical membrane-spanning segments of band 3 cluster such that all the charged residues of the amphipathic helices are facing the interior of an aqueous pore, as has been proposed for other transport proteins (18, 19). The positively charged residues of band 3 on the interior and exterior of the lipid bilayer would then act to funnel anions to the high-affinity binding site within this membranous pore (24). The region corresponding to hydrophobic peak $F$ is one of the few segments of the band 3 polypeptide that has been clearly demonstrated to span the membrane (8). Furthermore, this region is flanked by the external glycosylation site at residue 671 (Fig. 2) and the region corresponding to hydrophobic peak $\mathrm{E}$, which has also been shown to span the membrane $(8,35)$. The effect of removing the membrane-spanning segment corresponding to hydrophobic peak $F$ on the folding pattern and topology of the band 3 polypeptide and ultimately on its anion transport activity remains to be established. However, the possibility exists that removal of this transmembrane segment results in a band 3 polypeptide which has lost its capacity to exchange anions.

\section{ACKNOWLEDGMENTS}

We gratefully thank Nancy Vogelaar for her assistance in plotting the hydrophobicity profiles. We also thank Jeffrey Stack, Nigel Burns, and Frank Sangiorgi for their comments on the manuscript.

This work was supported by Public Health Service grants AG06078A and HL35801A from the National Institutes of Health and by grant DCB8215658 from the National Science Foundation. J. V. Cox was supported by a postdoctoral fellowship from the American Heart Association, Greater Los Angeles Affiliate.

\section{LITERATURE CITED}

1. Appell, K. C., and P. S. Low. 1981. Partial structural characterization of the cytoplasmic domain of the erythrocyte membrane protein, band 3. J. Biol. Chem. 256:11104-11111.

2. Aviv, H., and P. Leder. 1972. Purification of biologically active globin mRNA by chromatography on oligothymidylic acidcellulose. Proc. Natl. Acad. Sci. USA 69:1408-1412.

3. Bennett, V., and P. J. Stenbuck. 1979. The membrane attachment protein for spectrin is associated with band 3 in human erythrocyte membranes. Nature (London) 280:468-473.

4. Benton, W. D., and R. W. Davis. 1977. Screening $\lambda$ gt recombinant clones by hybridization to single plaques in situ. Science 196:180-182.

5. Berk, A. J., and P. A. Sharp. 1977. Sizing and mapping of early adenovirus mRNAs by electrophoresis of S1-endonuclease digested hybrids. Cell 12:721-732.

6. Bjerrum, P. J. 1983. Identification and location of amino acid residues essential for anion transport in red cell membranes, $p$. 107-115. In E. Quagliariello and F. Palmieri (ed.), Structure and function of membrane proteins. Elsevier Biomedical Press, Amsterdam.

7. Blikstad, I., W. J. Nelson, R. T. Moon, and E. Lazarides. 1983. Synthesis and assembly of spectrin during avian erythropoiesis: stoichiometric assembly but unequal synthesis of $\alpha$ and $\beta$ spectrin. Cell 32:1081-1091.

8. Brock, C. J., M. J. A. Tanner, and C. Kempf. 1983. The human erythrocyte anion-transport protein. Partial amino acid sequence and a possible molecular mechanism for anion exchange. Biochem. J. 213:577-586.

9. Capetanaki, Y. G., J. Ngai, C. Flytzanis, and E. Lazarides. 1983. Tissue-specific expression of two mRNA species transcribed from a single vimentin gene. Cell 35:411-420.

10. Cassoly, R., and J. M. Salhany. 1983. Spectral and oxygen release kinetic properties of hemoglobin bound to the cytoplasmic fragment of band 3 in solution. Biochim. Biophys. Acta 745:134-139.

11. Cohen, C. M., and C. Korsgren. 1985. Purification and properties of red cell band 4.2. J. Cell. Biol. 101:192a.

12. Cox, J. V., R. T. Moon, and E. Lazarides. 1985. Anion transporter: highly cell-type-specific expression of distinct polypeptides and transcripts in erythroid and nonerythroid cells. J. Cell. Biol. 100:1548-1557.

13. Cox, J. V., J. H. Stack, and E. Lazarides. 1986. Erythroid anion transporter assembly is mediated by a developmentally regulated recruitment onto a preassembled membrane cytoskeleton. J. Cell. Biol. 105:1405-1416.

14. Crandall, E. D., S. J. Mathew, R. S. Fleischer, H. I. Winter, and A. Bidani. 1981. Effects of inhibition of $\mathrm{RBC} \mathrm{HCO}_{3}{ }^{-} / \mathrm{Cl}^{-}$ exchange on $\mathrm{CO}_{2}$ excretion and downstream $\mathrm{pH}$ disequilibrium in isolated rat lungs. J. Clin. Invest. 68:853-862.

15. Demuth, D. R., L. C. Showe, M. Ballantine, A. Palumbo, P. J. Fraser, L. Cioe, G. Rovera, and P. J. Curtis. 1986. Cloning and structural characterization of a human nonerythroid band 3-like protein. EMBO J. 5:1205-1214.

16. Drenckhahn, D., K. Zinke, U. Schauer, K. C. Appell, and P. S. Low. 1984. Identification of immunoreactive forms of human erythrocyte band 3 in nonerythroid cells. Eur. J. Cell. Biol. 34:144-150.

17. Drickamer, L. K. 1976. Fragmentation of the 95,000-dalton transmembrane polypeptide in human erythrocyte membranes. J. Biol. Chem. 251:5115-5123.

18. Eisenberg, D. 1984. Three-dimensional structure of membrane and surface proteins. Annu. Rev. Biochem. 53:595-624.

19. Finer-Moore, J., and R. M. Stroud. 1984. Amphipathic analysis and possible formation of the ion channel in the acetylcholine receptor. Proc. Natl. Acad. Sci. USA 81:155-159.

20. Fukuda, M., Y. Eshdat, G. Tarone, and V. T. Marchesi. 1978. Isolation and characterization of peptides derived from the cytoplasmic segment of band 3, the predominant intrinsic membrane protein of the human erythrocyte. J. Biol. Chem. 253:2419-2438.

21. Hargreaves, W. R., K. N. Giedd, A. Verkliej, and D. Branton. 1980. Reassociation of ankyrin with band 3 in erythrocyte membranes and lipid vesicles. J. Biol. Chem. 255:11965-11972.

22. Jay, D. G. 1983. Characterization of the chicken erythrocyte anion exchange protein. J. Biol. Chem. 258:9431-9436. 
23. Jay, D. G. 1986. The glycosylation site of band 3, the human erythrocyte anion-exchange protein. Biochemistry 25:554-556.

24. Jay, D., and L. Cantley. 1986. Structural aspects of the red cell anion exchange protein. Annu. Rev. Biochem. 55:511-538.

25. Jennings, M. L., M. P. Anderson, and R. Monaghan. 1986. Monoclonal antibodies against human erythrocyte band 3 protein. J. Biol. Chem. 261:9002-9010.

26. Kaul, R. K., P. S. N. Murthy, A. G. Reddy, T. L. Steck, and H. Kohler. 1983. Amino acid sequence of the N-terminal 201 residues of human erythrocyte membrane band 3. J. Biol. Chem. 258:7981-7990.

27. Kay, M. M. B., C. M. Tracey, J. R. Goodman, J. C. Cone, and P. S. Bassel. 1983. Polypeptides immunologically related to band 3 in nucleated somatic cells. Proc. Natl. Acad. Sci. USA 80: 6882-6886.

28. Knauf, P. A. 1979. Erythrocyte anion exchange and the band 3 protein. Transport kinetics and molecular structure. Curr. Top. Membr. Transp. 12:249-263.

29. Kopito, R. R., M. Andersson, and H. F. Lodish. 1987. Structure and organization of the murine band 3 gene. J. Biol. Chem. 262: 8035-8040.

30. Kopito, R. R., and H. F. Lodish. 1985. Primary structure and transmembrane orientation of the murine anion exchange protein. Nature (London) 316:234-238.

31. Kozak, M. 1986. Point mutations define a sequence flanking the AUG initiator codon that modulates translation by eukaryotic ribosomes. Cell 44:283-292.

32. Kyte, J., and R. F. Doolittle. 1982. A simple method for displaying the hydropathic character of a protein. J. Mol. Biol. 157:105-132.

33. Laemmli, U. K. 1970. Cleavage of structural proteins during assembly of the head of bacteriophage T4. Nature (London) 227:680-685.

34. Low, P. S., M. A. Westfall, D. P. Allen, and K. C. Appell. 1984. Characterization of the reversible conformational equilibrium of the cytoplasmic domain of the erythrocyte membrane band 3 . J. Biol. Chem. 259:13070-13076.

35. Mawby, W. J., and J. B. C. Findlay. 1983. Characterization and partial sequence of di-iodosulphophenyl isothiocyanate-binding protein from human erythrocyte anion-transport protein. Biochem. J. 205:465-475.

36. Melton, D. A., P. A. Krieg, M. R. Rebagliati, T. Maniatis, K. Zinn, and M. R. Green. 1984. Efficient in vitro synthesis of biologically active RNA and RNA hybridization probes from plasmids containing a bacteriophage SP6 promoter. Nucleic Acids Res. 12:7035-7056.

37. Moon, R. T., J. Ngai, B. J. Wold, and E. Lazarides. 1985 Tissue-specific expression of distinct spectrin and ankyrin transcripts in erythroid and nonerythroid cells. J. Cell. Biol. 100: 152-160.

38. Murthy, S. N. P., T. Liu, R. K. Kaul, H. Kohler, and T. L. Steck. 1981. The aldolase-binding site of the human erythrocyte membrane is at the $\mathrm{NH}_{2}$ terminus of band 3. J. Biol. Chem. 256:
11203-11208.

39. Ngai, J., J. H. Stack, R. T. Moon. and E. Lazarides. 1987. Regulated expression of multiple chicken erythroid membrane skeletal protein 4.1 variants is governed by differential RNA processing and translational control. Proc. Natl. Acad. Sci. USA 84:4432-4436.

40. Pasternack, G. R., R. A. Anderson, T. L. Leto, and V. T. Marchesi. 1985. Interactions between protein 4.1 and band 3: an alternative binding site for an element of the membrane cytoskeleton. J. Biol. Chem. 260:3676-3683.

41. Rethmeier, R. A. F. 1979. Fragmentation of the band 3 polypeptide from human erythrocyte membranes. J. Biol. Chem. 254: 3054-3060.

42. Rigby, P. W. J., M. Dieckmann, C. Rhodes, and P. Berg. 1977. Labeling deoxyribonucleic acid to high specific activity in vitro by nick translation with DNA polymerase I. J. Mol. Biol. 113: 237-251.

43. Sanger, F., S. Nicklen, and A. R. Coulson. 1977. DNA sequencing with chain terminating inhibitors. Proc. Natl. Acad. Sci. USA 74:5463-5467.

44. Steck, T. L. 1974. The organization of proteins in the human red cell membrane. J. Cell. Biol. 62:1-19.

45. Steck, T. L., J. J. Koziarz, M. K. Singh, G. Reddy, and H. Kohler. 1978. Preparation and analysis of seven major topographically defined fragments of band 3 , the predominant transmembrane polypeptide of human erythrocyte membranes. Biochemistry 17:1216-1222.

46. Steck, T. L., B. Ramos, and E. Strapazon. 1976. Proteolytic dissection of band 3, the predominant transmembrane polypeptide of the human erythrocyte membrane. Biochemistry 15: 1154-1161.

47. Tsai, I.-H., S. N. P. Murthy, and T. L. Steck. 1982. Effect of red cell membrane binding on the catalytic activity of glyceraldehyde-3-phosphate dehydrogenase. J. Biol. Chem. 257:14381442 .

48. Weinstein, R. S., J. K. Khodadad, and T. L. Steck. 1978. Fine structure of the band 3 protein in human red cell membranes. Freeze-fracture studies. J. Supramol. Struct. 8:325-335.

49. Weislander, L. 1979. A simple method to recover intact high molecular weight RNA and DNA after electrophoretic separation in low gelling temperature agarose gels. Anal. Biochem. 98: 305-309.

50. Wieth, J. O., and P. J. Bjerrum. 1982. Titration of transport and modifier sites in the red cell anion transport system. J. Gen. Physiol. 79:253-282.

51. Wieth, J. O., P. J. Bjerrum, and C. L. Borders. 1982. Irreversible inactivation of red cell chloride exchange with phenylglyoxal, an arginine-specific reagent. J. Gen. Physiol. 79:283-312.

52. Yu, J., and T. L. Steck. 1975. Associations of band 3, the predominant polypeptide of the human erythrocyte membranes. J. Biol. Chem. 250:9176-9184.

53. Zaki, L. 1981. Inhibition of anion transport across red blood cells with 1,2-cyclohexanedione. Biochem. Biophys. Res. Commun. 99:243-251. 